\title{
Editorial
}

Die vier Beiträge dieser Ausgabe kommen aus den Gebieten der Wirtschaftsinformatik, der Unternehmensführung, der Wirtschaftsprüfung und der Bankwirtschaft.

\section{Fortlaufendes Datenqualitätsmanagement}

Zu Beginn ihres Beitrags weisen Heinrich und Klier darauf hin, dass wachsende Datenbestände, der zunehmende Einsatz von Data Warehouse-Systemen sowie ein effektives Kundenbeziehungsmanagement fortlaufende Maßnahmen zur Sicherstellung und Verbesserung der Qualität von Daten in Unternehmungen erforderlich machen. Diese vorweg sehr plausible Überlegung wird durch die empirischen Befunde gestützt, welche die Autoren zusammentragen. Demnach kann man davon ausgehen, dass 15 bis 20 Prozent der Datenbestände in Unternehmungen fehlerhaft sind, rund 70 Prozent der Marketingleiter sich darüber beklagen, dass Kunden unzufrieden auf eine schlechte Datenqualität im Hinblick auf Adressen und Information reagieren und - wohl auch insbesondere deswegen - 50 Prozent der Budgets für Data Warehouse-Projekte regelmäßig für das Datenqualitätsmanagement verwendet werden. In den USA wird der gesamtwirtschaftliche Schaden aufgrund mangelhafter Daten sogar auf insgesamt 600 Milliarden Dollar pro Jahr geschätzt. Effektives Datenmanagement im Hinblick auf die Qualitätskriterien Vollständigkeit, Korrektheit, Konsistenz und Aktualität erfordert aber ebenfalls Wirtschaftlichkeitsüberlegungen, die Aufschluss darüber geben sollen, wie groß der Umfang und wann die Zeitpunkte einer Investition in das Datenqualitätsmanagement sein sollen. Zu diesem Zweck formulieren die Verfasser ein mehrperiodiges quantitatives Entscheidungsmodell auf der Grundlage des Kapitalwertansatzes, wobei die erwarteten Einzahlungen aufgrund eines Qualitätsmanagements den Kosten des Datenqualitätsmanagements gegenübergestellt werden. Mithilfe der mathematischen Simulation auf der Basis eines numerischen Optimierungsverfahrens sollen Erkenntnisse über Indikatoren für ein fortlaufendes Datenqualitätsmanagement gewonnen werden. Die praktische Anwendung erfolgt für eine Fallstudie eines großen deutschen Mobilfunkanbieters. Hieraus lassen sich Handlungsempfehlungen zur Planung des Datenqualitätsmanagements ableiten, die sich gleichermaßen auf informationstechnische und ökonomische Aspekte beziehen. Die Ergebnisse der Simulation mithilfe einer Sensitivitätsanalyse, in welche 13 Modellparameter einbezogen werden, geben schließlich Auskunft darüber, inwieweit Umfang und Zeitpunkt der Investitionen - und ob diese dann gegebenenfalls im Zeitablauf gleich bleiben, fallen oder steigen sollen - von dem Umfang der Datenmenge, der Fülle der Basistransaktionen mit den Kunden sowie dem Zustand der Datenqualität determiniert werden. Diese Ergebnisse werden jedoch stark dadurch beeinflusst, welchen Wert der kundensegmentspezifische Parameter annimmt, der zum Ausdruck bringen soll, inwieweit ein Kundentyp die verbesserte Datenqualität im Kontakt mit dem Unternehmen schätzt, wie der Wirkungsgrad der Verbesserungsmaßnahmen eingestuft wird, in welchem Umfang die Maßnahmen von dem jeweiligen Kundentyp honoriert werden und wie stark sich die Grenzkosten bei zunehmender Maßnahmenintensität verändern.

\section{Steuerung mittelständischer Unternehmen}

Schachner, Speckbacher und Wentges untersuchen in ihrer Studie, welchen Einfluss die Unternehmensgröße, der Anteil der Eigentümerfamilie am Unternehmen sowie der Tatbestand, dass Familienmitglieder in der Geschäftsführung mitwirken, auf das Zielsystem, die 
Entscheidungsdelegation und die Implementierung strategischer Zielvorgaben in Form von Kennzahlen in mittelständischen Unternehmen haben. Die Einbeziehung des Aspektes des Anteils der Eigentümerfamilie und ihrer Mitwirkung in der Geschäftsführung sollen insbesondere darüber Aufschluss geben, inwieweit hierdurch widersprüchliche Befunde zu Größeneffekten der Unternehmungen erklärbar werden. Bisherige Berichte in der Literatur können in der Weise grob skizziert werden, dass in Unternehmungen, an denen die Eigentümerfamilie mehr als 50 Prozent der Anteile besitzt und welche ausschließlich von Familienmitgliedern geführt werden, unabhängig von der Unternehmensgröße weniger Entscheidungsdelegation und eine geringere Verwendung von Kennzahlensystemen stattfindet. Je mehr jedoch familienfremde Personen in die Geschäftsführung einbezogen werden, desto mehr werden formalisierte Steuerungssysteme benutzt und bei steigender Unternehmensgröße zentrale Führungsstrukturen angewandt. Zum Test der von den Autoren formulierten Hypothesen im Hinblick auf das Zielsystem, die dezentrale Steuerung und die Verwendung von Kennzahlensystemen sind 1400 kleinere und mittlere Unternehmungen aus Österreich und dem süddeutschen Raum sowie zusätzlich weitere 100 Unternehmen aus dem ATX und dem MDAX schriftlich befragt wurden, wovon schließlich 210 Antworten verwertbar waren. Auch wenn die empirischen Ergebnisse zunächst zeigten, dass 51 Prozent der Unternehmungen statt des finanziellen Erfolgs andere Ziele im Hinblick auf die Verbesserung der Beziehung zu Mitarbeitern, Kunden und Lieferanten an die erste Stelle ihres Zielsystems setzten, so konnte die Hypothese, dass Familienbetriebe Stakeholder-orientierte Zielgrößen für wichtiger halten als Nichtfamilienbetriebe nicht bestätigt werden. Dagegen konnte die Hypothese, dass Familienbetriebe geringere Eigenkapitalrenditen fordern, nicht abgelehnt werden. Die geforderte Eigenkapitalrendite wird wohl bei steigender Unternehmensgröße höher, dagegen werden die finanziellen Zielsetzungen nicht wichtiger. Für ausschließlich eigentümergeführte Unternehmungen bestätigte sich, dass in Familienbetrieben weniger Entscheidungsdelegation stattfindet. Ebenfalls konnte keine Abnahme der Zentralisierung bei steigender Unternehmensgröße nachgewiesen werden. Weiterhin lässt sich die Behauptung nicht stützen, dass mit steigender Unternehmensgröße formalisierte Steuerungssysteme in klein- und mittelständischen Unternehmungen zunehmen. Familienbetriebe machen davon im Allgemeinen weniger Gebrauch als Nichtfamilienbetriebe.

\section{Nachhaltigkeitsberichterstattung}

Nach den Unternehmenskrisen der Vergangenheit hatte sich die Meinung ausgebreitet, dass Prüfungsberichte alleine nicht mehr ausreichen, über die tatsächliche Einschätzung des Unternehmens hinreichende Auskünfte zu vermitteln, sondern dass die Unternehmen ihrer im Allgemeinen breiteren Verantwortung gerecht werden müssten und über den Erfolg, den sie erzielen, durch Nachhaltigkeitsberichte informieren sollen. Solche Nachhaltigkeitsberichte beziehen sowohl ökonomische als auch ökologische und soziale Größen mit ein, welche das unternehmerische Handeln beeinflussen und dazu führen sollen, Ressourcen für künftige Generationen zu erhalten. Quick und Knocinski versuchen, durch ihre Studie die Qualität deutscher Nachhaltigkeitsberichte gemäß der Grundsätze des GRI (Global Reporting Initiative) zu beurteilen. Im Rahmen der generellen Prinzipien Wirtschaftlichkeit, Transparenz, Dialogorientierung und Überprüfbarkeit sollen dabei die Art, Qualität und Verwertbarkeit der durch bestehende Nachhaltigkeitsberichte gegebenen Informationen eingeschätzt werden. Die Befunde werden dann mit Hilfe eines ScoringModells zu Gütemaßen aggregiert. Grundlage sind 26 Berichte von DAX30- und MDAXUnternehmungen, die von den Autoren nach 15 Oberkriterien eingestuft werden. Dabei 
werden sowohl allgemeine Angaben zur Unternehmung wie auch spezifische Antworten $\mathrm{zu}$ ökonomischen, sozialen und ökologischen Aspekten bezüglich der Einhaltung der Grundsätze der Berichterstattung nach GRI beurteilt. Bei der Auswertung der Unterlagen kommen die Verfasser zu dem Ergebnis, dass 50\% der überprüften Nachhaltigkeitsberichte sich auf GRI-Leitlinien beziehen. Im Bereich der allgemeinen Angaben wird ein Erreichungsgrad der Einhaltung der Prinzipien von $71 \%$ erzielt. Dagegen zeigen die Erreichungsgrade im Hinblick auf die spezifischen Angaben kaum zufrieden stellende Werte. Den ökonomischen Leitlinien des GRI wird durchschnittlich nur durch 14\% der Unternehmungen im Rahmen ihrer Nachhaltigkeitsberichterstattung entsprochen, wohingegen sich bei den sozialen Angaben ein Erreichungsgrad von 38\% und bei den ökologischen Berichtspunkten ein Erfüllungsgrad von $40 \%$ zeigt. Wenn die Nachteile der Nachhaltigkeitsberichterstattung, die vornehmlich in ihren Kosten und der durch sie hervorgerufenen öffentlichen Kritik am Unternehmen liegen, auch nicht verkannt werden, so sehen die Autoren jedoch deren Chancen darin, dass sie helfen kann, Kosten des Unternehmens zu senken, das Risiko zu mindern, Wettbewerbsvorteile zu generieren sowie die Unternehmensorganisation zu verbessern.

\section{Bankinterne Ratingverfahren als Ansatzpunkte zur Risikooptimierung}

In die Entscheidung der Kreditvergabe von Banken auf der Grundlage bankinterner Ratingverfahren gehen subjektive Bewertungen der Kreditbetreuer im Hinblick auf qualitative Unternehmensfaktoren ein, die zu Verzerrungen und Fehleinschätzungen bei der Beurteilung und Auswahl der Kreditnehmer führen können, wodurch letztlich der Erfolg der Bank maßgeblich beeinflusst wird. Hofmann analysiert nun mithilfe eines Principal-AgencyAnsatzes, inwieweit die bankinternen Ratingverfahren dazu genutzt werden können, geeignete Anreizsysteme für Kreditbetreuer zu installieren, die zu risikominderndem Verhalten der Kreditbetreuer führen und dadurch zugleich Konflikte zwischen dem Bankmanagement als Prinzipal und dem Kreditbetreuer als Agent weitgehend zu vermeiden helfen. Notwendigkeiten zur Ausgestaltung von Anreizsystemen für Kreditbetreuer, um deren potentielle Informationsvorteile über die Kreditnehmer im Sinne der Banken durch ein anreizkompatibles Entlohnungssystem zu nutzen, ergeben sich unmittelbar aus den Resultaten einer empirischen Studie, welche der Autor der modelltheoretischen Analyse vorangestellt hat. Die Befragung von 500 kleineren und mittleren deutschen Kreditinstituten, von denen schließlich 64 auswertbare Beantwortungen zurücksandten, zeigt, dass das Gewicht subjektiver, qualitativer Unternehmensfaktoren bei der Entscheidung der Kreditvergabe zwischen 0 und 60\% ausmachen kann und von Bank zu Bank sehr stark schwankt. Dabei wird nur in $44 \%$ der ausgewerteten Kreditinstitute eine Beteiligung der Kreditbetreuer an dem von ihnen mitbeeinflussten Selektions- und Betreuungserfolg vorgesehen, wobei sich diese nur in 5\% der Fälle unmittelbar in der Entlohnung niederschlägt - aber nur in 46\% der Kreditinstitute ist das Ergebnis des jeweiligen Kredits direkter Maßstab für die Entlohnung. So ist insgesamt in den untersuchten kleinen und mittelgroßen Kreditinstituten das Bewusstsein einer erfolgsorientierten Steuerung der Kreditbetreuer nur gering ausgeprägt. Welche Vorteile aber eine anreizeffiziente Mitarbeitersteuerung für Prinzipal und Agent in Anlehnung an das bankinterne Ratingverfahren haben kann, untersucht der Verfasser anschließend in seiner agency-theoretischen Modellanalyse. Der Erfolg der Anstrengungen des Kreditbetreuers, durch verbesserte Marktkenntnisse und deren Nutzung bei der Kreditvergabe das Risiko des Kredits - gemessen an seiner Ertragsvarianz - zu reduzieren, soll Grundlage für die Ertragsbeteiligung des Kreditbetreu- 
ers sein. Für Mitarbeiter und Bankmanagement wird unterstellt, dass sie risikoavers sind und negative exponentielle Nutzenfunktionen besitzen. Analytische Überlegungen zur optimalen Risikoaufteilung bei vollkommener Information (First-Best-Lösung) und bei bestehenden Informationsasymmetrien zwischen Bankmanagement und Kreditbetreuer (Second-Best-Lösung) zeigen, dass der Kreditbetreuer bei vollkommener Information ein höheres Anstrengungsniveau zur risikomindernden Kreditvergabe an den Tag legt, der erfolgsabhängige variable Teil seiner Vergütung jedoch bei Informationsasymmetrien höher ausfallen muss. Im Übrigen lassen sich durch das Modell sehr schön die Interdependenzen zwischen dem Grad der Marktkenntnis des Kreditbetreuers, dem jeweiligen Status der Ratingtechnologie, der Risikoeinstellung von Prinzipal und Agent sowie der Integration der Marktfolge durch das Bankmanagement in den Entscheidungsprozess studieren; sie zeigen, welche Auswirkungen die Risikotragfähigkeit der Akteure auf das Risikoteilungsproblem hat. In jedem Fall zahlen sich Verbesserungen der Ratingtechnologie und der Marktkenntnis für die Akteure aus.

\section{Das Juli/August-Heft}

Das nächste Heft der ZfB wird eine Doppelausgabe sein, da die bisher bereits abgedruckten Beiträge und die für das Doppelheft vorgesehenen Artikel den geplanten Umfang an Heftseiten einschließlich der August-Ausgabe der ZfB übersteigen.

In der nächsten Ausgabe gelangen fünf Arbeiten zum Abdruck. Sie können den Gebieten der Allgemeinen Betriebswirtschaft, der Investitionstheorie, der Unternehmensbewertung, der Wirtschaftsprüfung sowie des Marketings zugeordnet werden.

Albach berichtet über die Schwerpunkte und Ergebnisse von rund 40 Jahren Forschung an den vier betriebswirtschaftlichen Lehrstühlen der Rechts- und Staatswissenschaftlichen Fakultät der Universität Bonn von Beginn der sechziger bis zum Ende der neunziger Jahre des letzten Jahrhunderts. Die Synopse dokumentiert sehr anschaulich die Entwicklungen zu einer empirischen Theorie der Unternehmung.

Mölls und Schild untersuchen den Einfluss der minimalen Investitionsrate in sequentiellen Investitionsprojekten. Hierzu wird ein Entscheidungsmodell auf der Grundlage des Realoptionsansatzes formuliert, dessen Modellierung auf partiellen Differenzialgleichungen basiert.

Reichling, Spengler und Vogt nehmen in ihrem Beitrag zu Sicherheitsäquivalenten, Wertadditivität und Risikoneutralität Stellung. Sie zeigen, dass die Unternehmensbewertung mit Hilfe der Methode der Sicherheitsäquivalente unter der Annahme der Wertadditivität konstante absolute wie auch konstante relative Risikoaversion impliziert. Da beides nur bei Risikoneutralität erfüllt ist, wird eine Bestimmung des Unternehmenswertes nach der Sicherheitsäquivalentmethode überflüssig.

Simons und Biskup diskutieren die Frage, ob ein Bedarf nach Dritthaftung des gesetzlichen Jahresabschlussprüfers besteht. Unabhängig von unterschiedlichen rechtlichen Positionen und deren Veränderung, die in Deutschland im Vergleich zur USA auszumachen sind, analysieren die Autoren spieltheoretisch, unter welchen Bedingungen ein solcher Bedarf nach Dritthaftung auftritt.

Den Einfluss von Meinungsführern auf den Erfolg von hedonischen Produkten untersuchen Clement, Proppe und Sambeth. Insbesondere wird analysiert, inwieweit sich Aussagen von Meinungsführern aus dem literarischen Quartett auf den Erfolg von Büchern auswirken.

Günter Fandel

Editor-in-Chief 